\title{
Localization with Magnetic Field Distortions and Simultaneous Magnetometer Calibration
}

\author{
Benjamin Siebler, Member, IEEE, Stephan Sand, Senior Member, IEEE, Uwe D. Hanebeck, Fellow, IEEE
}

\begin{abstract}
Magnetic field localization utilizes position dependent and time persistent distortions of the earth magnetic field. These distortions are introduced by stationary ferromagnetic material in the environment and can be stored in a map to enable localization. Estimating the position of a magnetometer with these distortions requires a calibration of the sensor to enable the matching of the measurements to the map. Typically, the calibration is performed in a prior step and requires specific maneuvers like sensor rotations in a homogenous field. The goal of the maneuvers is to render the calibration parameters observable. For heavy platforms, e.g., cars, trains and driverless transport systems in factories, performing special maneuvers is cumbersome or even impossible. In addition they operate in an environment with an inhomogeneous magnetic field. To address this issue, this paper proposes a novel method that exploits the magnetic field distortions to render the calibration parameters observable. To simplify the calibration process, the calibration parameters are estimated simultaneously with the position of the platform. The method employs a Rao-Blackwellized particle filter that reduces the computational complexity and enables real time processing. The feasibility of the method is shown in an evaluation with measurements of a magnetometer mounted on a model train. The results show a high accuracy of the position and calibration parameter estimation.
\end{abstract}

Index Terms-Magnetometer calibration, magnetic field localization, Rao-Blackwellized particle filtering

\section{INTRODUCTION}

$\mathbf{R}$ OBUST and accurate localization is important for the automation of traffic and logistics and therefore an integral part of smart cities. Nevertheless, providing localization in all relevant environments is still a challenge. Even outside, where global navigation satellite systems (GNSS), e.g., GPS and Galileo can be received, the availability and accuracy of the obtained position is often degraded due to shadowing and multipath propagation. To achieve a high availability it is therefore vital to combine GNSS with other sensors. Typical sensors combined with GNSS are inertial measurement units (IMUs) and odometers. Both sensors only provide relative position information with an inherent drift, which limits the time for which high position accuracy can be maintained during GNSS outages. For indoor environments the situation is even more challenging because GNSS signals are completely blocked. Hence, many possible alternatives are discussed where the usability of the different approaches depends on the user. For

Benjamin Siebler and Stephan Sand are with the German Aerospace Center (DLR), Wessling, Bavaria 82234 Germany (e-mail: benjamin.siebler@dlr.de and stephan.sand@dlr.de).

Uwe D. Hanebeck is with the Karlsruhe Institute of Technology (KIT) Intelligent Sensor-Actuator-Systems Laboratory, Karlsruhe, Baden-Wuerttemberg 76021 Germany (e-mail: uwe.hanebeck@kit.edu).

Manuscript received Month Day, Year; revised Month Day, Year. a pedestrian using IMUs attached to the body is a popular approach. To limit the drift the IMUs are regularly recalibrated during certain phases of the pedestrian's movement cycle [1]. Another popular approach, applicable not only to pedestrians, is the use of signals of opportunity ( $\mathrm{SoO})$. SoO are signals that can be used for but are not dedicated to localization. Examples of such signals are radio signals from communications equipment, e.g., Wi-Fi access points. A variety of methods for SoO localization using different measurement methods like received signal strength fingerprints or time delay measurement are considered [2] and are sometimes combined with simultaneous localization and mapping (SLAM) methods [3] to additionally estimate the position of the signal sources. In this paper, the focus is on localization with local distortions of the earth magnetic field, which can be considered as a SoO. In buildings, steel reinforced concrete and steel beams create a strongly varying magnetic field suitable for localization [4]. On roads and railway tracks, the distortions are caused, e.g., by buildings, and lamp and traffic light posts. The feasibility of magnetic localization has been shown indoors for pedestrians and wheeled robots and outdoors for cars, airplanes, and trains [5]-[17]. In [18], [19] we proposed two algorithms for magnetic field based train localization. In accordance with the before mentioned literature, we implicitly assumed calibrated magnetometers. This guarantees that the magnetometer measurements fit to the values in the map. In practice, the calibration of magnetometers in a train, or for heavy platforms in general, is not easily obtained. Standard calibration methods require the sensor and the platform, on which it is mounted, to rotate in a homogenous magnetic field [20]-[24]. In [12], the authors calibrate the magnetometers mounted in a car by driving circles in a homogenous field and then applying a modified ellipsoid fitting technique. Due to the limited degrees of freedom during the calibration maneuvers the parameters of the vertical axis are not observable. An alternative is to apply a known but varying magnetic field on the platform and the sensor. Both possibilities are cumbersome to use when the platforms have a large size or mass.

We therefore propose a novel method allowing the estimation of all calibration parameters. Instead of rotating the sensor in a homogenous field, the magnetometer moves through an inhomogeneous magnetic field that is known from a map. When the distortions excite the sensor sufficiently all parameters are observable. However, to know the current magnetic field value, the platform position must be known and to estimate the position from the magnetic field, the calibration is required. To address this issue, a joint estimation problem is formulated and solved with a Rao-Blackwellized particle filter. 


\section{Sensor Calibration}

In this section the sensor calibration model is introduced and it is shown how an inhomogeneous magnetic field can be used to estimate the calibration parameters.

\section{A. Sensor Model}

An ideal calibration removes the effects from surrounding material on the magnetometer measurements. The calibration can be considered as a function that maps the true magnetic vector field in the navigation frame $\mathbf{z}^{\mathrm{n}} \in \mathbb{R}^{3}$, e.g., the earth magnetic field, to the magentic field in the sensors body frame $\mathbf{z}^{\mathrm{b}} \in \mathbb{R}^{3}$ that is measured with a magnetometer triad. A common approach is to assume a linear model, e.g., [20][24]. The linear model considers two effects due to material in the vicinity of the sensor

- Hard iron effects that introduce a constant offset due to a constant magnetization.

- Soft iron effects that rotate and scale external fields applied to the sensor.

In addition the sensor hardware potentially contains errors that are also covered by a linear model

- Non-orthogonality of the sensor axes.

- Scale-errors due to different gains for each axis.

- Offsets leading to constant nonzero measurements in the absence of an external field.

Combining the different effects and errors lead to the linear model

$$
\mathbf{z}^{\mathrm{b}}=\mathbf{M}_{\text {scale }} \mathbf{M}_{\text {no }}\left(\mathbf{M}_{\text {soft }} \mathbf{R}_{\mathrm{n}}^{\mathrm{b}}(t) \mathbf{z}^{\mathrm{n}}+\mathbf{b}_{\text {hard }}\right)+\mathbf{b}_{\mathrm{o}}+\mathbf{n}
$$

where the matrices $\mathbf{M}_{\text {scale }}, \mathbf{M}_{\mathrm{no}}, \mathbf{M}_{\text {soft }}$ and $\mathbf{R}_{\mathrm{n}}^{\mathrm{b}}(t)$ account for the scaling and non-orthogonality of the axes, the rotation and scaling due to soft iron effects, and the rotation between sensor and navigation frame. The vectors $\mathbf{b}_{\text {hard }}$ and $\mathbf{b}_{\mathrm{o}}$ are the offsets due to hard iron effects and the sensor errors. The last term in (1) is the measurement noise $\mathbf{n}$.

For calibration, we assume that only the rotation matrix $\mathbf{R}_{\mathrm{n}}^{\mathrm{b}}(t)$ is time-variant due to the changing attitude of the platform on which the sensors are mounted. For calibration it is not necessary to estimate every part of (1) separately and therefore the model can be simplified to

$$
\mathbf{z}^{\mathrm{b}}=\mathbf{C R}_{\mathrm{n}}^{\mathrm{b}}(t) \mathbf{z}^{\mathrm{n}}+\mathbf{b}+\mathbf{n}
$$

During calibration the sensor is usually rotated. Furthermore, a known sensor attitude $\mathbf{R}_{\mathrm{n}}^{\mathrm{b}}(t)$ and magnetic field vector $\mathbf{z}^{\mathrm{n}}$ is assumed to estimate the calibration parameters. For a perfectly calibrated sensor that is rotated in a homogenous field the measurements describe a sphere with radius equal to the magnitude of the homogenous field. In contrast, measurements of an uncalibrated sensors form an ellipsoid. The calibration therefore maps an ellipsoid to a sphere. Since $\mathbf{R}_{\mathrm{n}}^{\mathrm{b}}(t)$ is often unknown, the calibration can also be performed only with the magnitude of magnetometer measurement $\left\|\mathbf{z}^{\mathrm{b}}\right\|$. If only the magnitude is used for calibration, the calibration contains a non-observable rotation. This is because the only knowledge used in the calibration is that measurements lie on the surface of a sphere with a certain radius. A multiplication of the calibrated measurements with an arbitrary rotation still results in a sphere and therefore is still a valid calibration.

\section{B. Sensor Calibration with Known Magnetic Field}

When it is not possible to rotate the platform the observability of the calibration parameters must be ensured by changing the external magnetic field, e.g., by moving the sensor in an inhomogeneous but known magnetic field. In contrast to other calibration approaches, the primary goal in this paper is not to calibrate the sensor so that it measures the true magnetic field and its physical dimension. Instead the goal is to calibrate the sensor with respect to the magnetic field stored in a map. The map itself can be recorded with an uncalibrated sensor. This is possible because the calibration model is linear and therefore the composition of models is again linear. So our calibration can be described basically as a relative calibration that enables us to use uncalibrated sensors to record a map and then calibrate every other magnetometer relative to that map. By not trying to find the true magnetic field, the whole mapping and localization process is simplified. This is not a limitation of the approach because if the map contains the true physical magnetic field also the calibration can be used to obtain measurements with the correct physical dimensions. The success of this approach strongly depends on the magnetic field, which the magnetometer measures during its movement. When there are too little distortions, the model parameters are not observable. This issue will be discussed in more detail in the following.

If the platform attitude and position is known the estimation of the parameters in (2) is trivial because for each measurement $\mathbf{z}^{\mathrm{b}}$ the counterpart $\mathbf{z}^{\mathrm{n}}$ is known from the map and the estimation problem becomes linear. This can be seen from writing (2) as a function of the calibration parameter vector $\boldsymbol{\theta}$

$$
\mathbf{z}^{\mathrm{b}}=\mathrm{h}\left(\mathbf{z}^{\mathrm{n}}\right) \boldsymbol{\theta}+\mathbf{n},
$$

with the measurement function $\mathrm{h}(\cdot)$

$$
\mathrm{h}\left(\mathbf{z}^{\mathrm{n}}\right)=\left[\begin{array}{ll}
\mathbf{I}_{3 \times 3} \otimes \mathbf{z}^{\mathrm{n} T} & \mathbf{I}_{3 \times 3}
\end{array}\right]
$$

and

$$
\boldsymbol{\theta}=\left[\begin{array}{llll}
\mathbf{c}_{1} & \mathbf{c}_{2} & \mathbf{c}_{3} & \mathbf{b}^{T}
\end{array}\right]^{T},
$$

where $\mathbf{I}_{3 \times 3}$ is the three dimensional identity matrix, $\otimes$ the Kronecker product, and $\mathbf{c}_{i}$ is the $\mathrm{i}$-th row of matrix $\mathbf{C}$. To be able to estimate $\boldsymbol{\theta}$ in (3) at least four measurements of a magnetometer triad are required. The simplest way to estimate $\boldsymbol{\theta}$ is to stack all $N_{\mathbf{z}}$ available magnetometer measurements into a single measurement vector of the dimension $3 \cdot N_{\mathbf{z}} \times 1$. This results in the system of linear equations

$$
\left[\begin{array}{c}
\mathbf{z}_{1}^{\mathrm{b}} \\
\vdots \\
\mathbf{z}_{N_{\mathbf{z}}}^{\mathrm{b}}
\end{array}\right]=\underbrace{\left[\begin{array}{c}
\mathrm{h}\left(\mathbf{z}_{1}^{\mathrm{n}}\right) \\
\vdots \\
\mathrm{h}\left(\mathbf{z}_{N_{\mathbf{z}}}^{\mathrm{n}}\right)
\end{array}\right]}_{\mathbf{D}} \boldsymbol{\theta}+\left[\begin{array}{c}
\mathbf{n}_{1} \\
\vdots \\
\mathbf{n}_{N_{\mathbf{z}}}
\end{array}\right] .
$$

Assuming white Gaussian measurement noise the maximum likelihood estimate of (6) is found by a, possibly weighted, least-squares estimator (LSE). The pseudo inverse of the 
design matrix $\mathbf{D}$ for the LSE only exists when at least four linearly independent measurements are available. In the presence of noise the achievable accuracy strongly depends on the collinearity of the data in the design matrix. If the data has strong correlations the accuracy will be low. This is the case, e.g., when the platform does not move considerably during the time the measurements are recorded.

When the position and attitude is known linear filter algorithms like the well known Kalman filter can be used as an alternative to the LSE to estimate the parameters. This is particularly helpful when the estimation is performed online in a recursive manner. The Kalman filter requires in addition to the measurement function (3) a model for the temporal evolution of the parameters $\boldsymbol{\theta}$. Assuming almost constant calibration parameters, the temporal behavior can be described by a time discrete random walk

$$
\boldsymbol{\theta}_{k}=\boldsymbol{\theta}_{k-1}+\mathbf{w}_{k-1}^{\theta}
$$

where the index $k$ represents the discrete time step and $\mathbf{w}_{k}^{\theta}$ is a small Gaussian process noise. The Kalman filter intrinsically accounts for the observability of the parameters. If the measurements are correlated and the Kalman filter is not already converged, the state covariance is large indicating large uncertainty in the estimation and vice versa. The Kalman filter not only accounts for the observability but also gives the lowest possible estimation error. This can be seen from its relation to the posterior Cramér-Rao lower bound (PCRLB). The PCRLB is a lower bound on the mean-square-error and therefore limits the performance of any Bayesian estimator [25]. Furthermore, the PCRLB can be seen as a soft metric for the observability of a dynamic system. For a linear system with additive Gaussian noise the Kalman filter is unbiased and exactly attains the bound and therefor the state covariance matrix is equal to the bound [25]. This property of the Kalman filter is important for the proposed simultaneous localization and calibration filter because in the update step the uncertainty of the estimated parameters is required.

\section{Simultaneous Localization And Sensor CALIBRATION}

The concept of particle filter based magnetic localization was already introduced, e.g., in [9], [13], [19]. For simplification, the sensors were calibrated beforehand or the map was recorded with the same setup that was used for localization rendering calibration unnecessary. In this section, the particle filter for localization is extended to simultaneously estimate the magnetometer calibration parameters based only on a map of the magnetic field and the magnetometer measurements.

\section{A. Problem Definition}

The map of the magnetic field $\mathrm{m}(\cdot)$ is a function that maps the position of the sensor to the magnetic vector at that position. With the map the state space model for the estimation problem can be formulated. All states regarding the translatory dynamics of the platform are lumped together in the vector $\mathbf{d}_{k}$ which at least contains the position and depending on the motion model also the speed and acceleration of the platform.
The attitude is represented by the vector $\mathbf{q}_{k}$ that can be adapted depending on the degree of freedom of the platform. For 2-D applications it might only contain the heading and for 3-D applications in addition pitch and roll angles. The vectors $\mathbf{d}_{k}$ and $\mathbf{q}_{k}$ form the pose $\mathbf{p}_{k}=\left[\begin{array}{ll}\mathbf{d}_{k}^{T} & \mathbf{q}_{k}^{T}\end{array}\right]^{T}$ of the platform. Besides $\mathbf{p}_{k}$ also the calibration parameter vector $\boldsymbol{\theta}_{k}$ is estimated. The combined state therefore is

$$
\mathbf{x}_{k}=\left[\begin{array}{lll}
\mathbf{d}_{k}^{T} & \mathbf{q}_{k}^{T} & \boldsymbol{\theta}_{k}^{T}
\end{array}\right]^{T}=\left[\begin{array}{ll}
\mathbf{p}_{k}^{T} & \boldsymbol{\theta}_{k}^{T}
\end{array}\right]^{T} .
$$

The generic state space model for the estimation problem is given by

$$
\begin{aligned}
\mathbf{x}_{k} & =\left[\begin{array}{c}
\mathrm{f}\left(\mathbf{p}_{k-1}, \mathbf{w}_{k-1}^{p}\right) \\
\boldsymbol{\theta}_{k-1}+\mathbf{w}_{k-1}^{\theta}
\end{array}\right] \\
\mathbf{z}_{k}^{\mathrm{b}} & =\mathbf{R}_{\mathrm{n}}^{\mathrm{b}}\left(\mathbf{q}_{k}\right) \mathrm{h}\left(\mathrm{m}\left(\mathbf{d}_{k}\right)\right) \boldsymbol{\theta}_{k}+\mathbf{n}_{k}
\end{aligned}
$$

with the system function $\mathrm{f}(\cdot)$, the measurement function $\mathrm{h}(\cdot)$ from (4) and the process noise of the pose $\mathbf{w}_{k}^{p}$ and parameters $\mathbf{w}_{k}^{\theta}$. The pose movement and attitude model $\mathrm{f}(\cdot)$ is not limited to linear functions. For simplicity, the process noise follows the Gaussian distributions $\mathbf{w}_{k}^{p} \sim \mathcal{N}\left(\mathbf{0}, \mathbf{Q}_{p}\right)$ and $\mathbf{w}_{k}^{\theta} \sim \mathcal{N}\left(\mathbf{0}, \mathbf{Q}_{\theta}\right)$. The choice of the pose noise covariance $\mathbf{Q}_{p}$ depends on the dynamics of the platform and must be chosen accordingly. The variance of the parameters is treated as a tuning factor. Theoretically the variance should be zero since the parameters are assumed constant but using a nonzero value enables the control of the convergence speed and the steady state behavior of the Kalman filter. A nonzero value also can be beneficial when the parameters slowly change over time, e.g., due to a temperature drift in the magnetometer. For the measurements additive white Gaussian noise $\mathbf{n}_{k} \sim \mathcal{N}\left(\mathbf{0}, \mathbf{R}_{k}\right)$ is assumed. The goal of the filter design is to obtain at each time step $k$ a point estimate of the state vector (8) conditioned on the complete measurement history

$$
\mathbf{z}_{1: k}^{\mathrm{b}}=\left[\begin{array}{lll}
\mathbf{z}_{1}^{\mathrm{b}^{T}} & \cdots & \mathbf{z}_{k}^{\mathrm{b}^{T}}
\end{array}\right]^{T} .
$$

To achieve this, the full posterior of $\mathbf{x}$ is estimated. From the posterior it is then possible to obtain different point estimates like the minimum mean-square error estimate (MMSEE) or the maximum a posteriori estimate.

\section{B. Particle Filter for Localization and Calibration}

The estimation of the posterior density is performed with a recursive Bayesian filter that is able to perform this task in real time. The closed-form analytical computation of the posterior is only feasible for a small class of system models like linear Gaussian systems. For these systems, the Kalman filter estimates the posterior efficiently. For nonlinear systems, a numerical approximation is required in most cases. For the particular estimation problem in this paper, the nonlinearities are due to the magnetic field map that is part of the measurement model and possibly the pose system model. Therefore, the posterior probability density function (PDF) is approximated with a particle cloud that represents the posterior with a set of $N_{p}$ weighted particles, where each particle $\mathrm{x}_{0: k}^{i}$ with $i \in\left\{1, \ldots, N_{p}\right\}$ represents a state trajectory hypothesis 
for the time steps 0 to $k$. This results in a Dirac mixture density

$$
p\left(\mathbf{x}_{0: k} \mid \mathbf{z}_{1: k}^{\mathrm{b}}, \mathrm{m}\right)=\sum_{i=1}^{N_{p}} w_{k}^{i} \delta_{\mathbf{x}_{0: k}^{i}}\left(\mathbf{x}_{0: k}\right),
$$

where $\delta$ is the Dirac measure

$$
\delta_{\mathbf{x}_{0: k}^{i}}\left(\mathbf{x}_{0: k}\right)= \begin{cases}1, & \text { if } \mathbf{x}_{0: k}=\mathbf{x}_{0: k}^{i} \\ 0, & \text { otherwise }\end{cases}
$$

and $w_{k}^{i} \geq 0$ the positive and normalized particle weights that sum up to one

$$
\sum_{i=1}^{N_{p}} w_{k}^{i}=1
$$

The particle filter is a generic filter that theoretically can handle all kinds of nonlinearities and the result converges to the true posterior when the number of particles goes to infinity. In reality the number of particles are constrained by the memory size, computational resources and time constraints in real time applications. To keep the number of particles small it is vital to also keep the state dimension small because a particle filter has to sample the whole state space. A higher state dimension therefore requires more particles to maintain the same particle density in each dimension. This is often called the curse of dimensionality. If the density is too low the posterior is not well represented and the filter most likely will diverge. For the state vector $\mathbf{x}$ in (8) the dimension is $\operatorname{dim}(\mathbf{p})+12$. The minimum dimension is therefore 13 when a 1-D position, e.g., of a train on a railway track is considered. Thus, for the case of only ten particles per dimension already $10^{13}$ particles would be required. This amount cannot be processed in real time with a reasonable update rate and memory requirement. To reduce the computational burden the structure of the problem is exploited. The problem contains linear substructures that can be estimated by a Kalman filter and therefore do not have to be sampled. This concept is known under different names like marginalized particle filter [26] or Rao-Blackwellized particle filter [27] and is useful for many nonlinear estimation problems which obey a certain structure. One popular example is the FastSLAM algorithm [28] for SLAM applications with many landmarks. In a marginalized particle filter the state vector $\mathbf{x}$ is decomposed into two parts $\mathrm{x}^{\mathrm{nl}}$ and $\mathrm{x}^{1}$. The decomposition requires that the state space model of $x^{1}$ conditioned on the measurements and $\mathrm{x}^{\mathrm{nl}}$ is linear and therefore analytically tractable. With the decomposed state the posterior density becomes

$$
p\left(\mathbf{x}_{k}^{1}, \mathbf{x}_{0: k}^{\mathrm{nl}} \mid \mathbf{z}_{1: k}^{\mathrm{b}}, \mathrm{m}\right)=\underbrace{p\left(\mathbf{x}_{k}^{\mathrm{l}} \mid \mathbf{x}_{0: k}^{\mathrm{nl}}, \mathbf{z}_{1: k}^{\mathrm{b}}, \mathrm{m}\right)}_{\text {linear }} p\left(\mathbf{x}_{0: k}^{\mathrm{nl}} \mid \mathbf{z}_{1: k}^{\mathrm{b}}, \mathrm{m}\right) .
$$

In (15) only the nonlinear part $p\left(\mathbf{x}_{0: k}^{\mathrm{nl}} \mid \mathbf{z}_{1: k}^{\mathrm{b}}, \mathrm{m}\right)$ has to be estimated with a particle filter. This reduces the sampled state space from $\operatorname{dim}\left(\mathbf{x}^{1}\right)+\operatorname{dim}\left(\mathbf{x}^{\mathrm{nl}}\right)$ to $\operatorname{dim}\left(\mathbf{x}^{\mathrm{nl}}\right)$. For the linear part, only the filter density at time step $k$ is estimated. For the estimation problem under consideration, the linear part $\mathbf{x}^{1}$ of the state vector $\mathbf{x}$ in (8) is the parameter vector $\boldsymbol{\theta}$ and the nonlinear parts is $\mathbf{p}$. The posterior therefore becomes

$$
p\left(\mathbf{p}_{0: k}, \boldsymbol{\theta}_{k} \mid \mathbf{z}_{1: k}^{\mathrm{b}}, \mathrm{m}\right)=\underbrace{p\left(\boldsymbol{\theta}_{k} \mid \mathbf{p}_{0: k}, \mathbf{z}_{1: k}^{\mathrm{b}}, \mathrm{m}\right)}_{\text {Kalman filter }} \underbrace{p\left(\mathbf{p}_{0: k} \mid \mathbf{z}_{1: k}^{\mathrm{b}}, \mathrm{m}\right)}_{\text {particle filter }},
$$

where the Kalman filter estimates of the calibration parameters are based on the linear model (7) and (10). In the following we will drop the map $\mathrm{m}(\cdot)$ and the superscript $b$ for the measurements to simplify the notation. The particle filter used in this paper is a sampling importance resampling (SIR) filter [29]. In the SIR filter the importance density, from which the samples of $\mathbf{p}$ are drawn, is the one step prediction density $p\left(\mathbf{p}_{k} \mid \mathbf{p}_{k-1}\right)$ which simplifies the weight update in the filter to

$$
w_{k}^{i} \propto w_{k-1}^{i} p\left(\mathbf{z}_{k} \mid \mathbf{p}_{0: k}^{i}, \mathbf{z}_{1: k-1}\right) .
$$

The evaluation of the likelihood in (17) is not directly possible because equation (10) is not defined without the parameter vector $\boldsymbol{\theta}$. Therefore, the likelihood is related to the parameter vector with a marginalization [30]

$$
\begin{array}{r}
p\left(\mathbf{z}_{k} \mid \mathbf{p}_{0: k}^{i}, \mathbf{z}_{1: k-1}\right)=\int p\left(\mathbf{z}_{k} \mid \mathbf{p}_{0: k}^{i}, \mathbf{z}_{1: k-1}, \boldsymbol{\theta}_{k}\right) \times \\
p\left(\boldsymbol{\theta}_{k} \mid \mathbf{p}_{0: k}^{i}, \mathbf{z}_{1: k-1}\right) d \boldsymbol{\theta}_{k} .
\end{array}
$$

In (18) the PDF $p\left(\boldsymbol{\theta}_{k} \mid \mathbf{p}_{0: k}^{i}, \mathbf{z}_{0: k-1}\right)$ is the prior Gaussian distribution of the parameters at time step $k$ predicted by the Kalman filter based on the trajectory of the $i$-th particle

$$
p\left(\boldsymbol{\theta}_{k} \mid \mathbf{p}_{0: k}^{i}, \mathbf{z}_{1: k-1}\right)=\mathcal{N}\left(\boldsymbol{\theta}_{k-1}^{i}, \mathbf{P}_{k-1}^{i}+\mathbf{Q}_{\boldsymbol{\theta}}\right) .
$$

In (19) the covariance matrix $\mathbf{P}_{k}^{i}=\mathbf{P}_{k-1}^{i}+\mathbf{Q}_{\boldsymbol{\theta}}$ is the predicted state covariance of the Kalman filter and $\boldsymbol{\theta}_{k-1}$ is the predicted parameter vector which is equal to the posterior mean of the previous step due to the random walk assumption in (7). The integral in (18) is therefore a convolution of the Gaussian prior PDF of the parameters with the Gaussian likelihood $p\left(\mathbf{z}_{k} \mid \mathbf{p}_{0: k}^{i}, \mathbf{z}_{1: k-1}, \boldsymbol{\theta}_{k}\right)$ which results in

$$
p\left(\mathbf{z}_{k} \mid \mathbf{p}_{0: k}^{i}, \mathbf{z}_{1: k-1}\right)=\mathcal{N}\left(\mathbf{z}_{k} ; \mathbf{H}_{k}^{i} \boldsymbol{\theta}_{k-1}^{i}, \boldsymbol{\Sigma}_{k}^{i}\right)
$$

with $\mathbf{H}_{k}^{i}=\mathbf{R}_{\mathrm{n}}^{\mathrm{b}}\left(\mathbf{q}_{k}^{i}\right) \mathrm{h}\left(\mathrm{m}\left(\mathbf{d}_{k}^{i}\right)\right)$ and the covariance matrix

$$
\boldsymbol{\Sigma}_{k}^{i}=\mathbf{R}_{k}+\mathbf{H}_{k}^{i} \mathbf{P}_{k}^{i} \mathbf{H}_{k}^{i^{T}} .
$$

The convolution of the two Gaussian distributions in (18) therefore is a Gaussian distribution where the measurement and parameter uncertainties are added up [30]. This means that the degree of observability of the parameter vector is included in the weight update by the state covariance matrix $\mathbf{P}_{k}^{i}$ of the Kalman filter. Algorithm 1 shows the pseudo code of one iteration of the marginalized particle filter in detail.

\section{EVALUATION}

The feasibility of the proposed approach is shown for a platform following a fixed trajectory, e.g., a train on a track [19] or a path following platform encountered in factories. This example is particular interesting because even partial calibration by driving a specific maneuver like in [13] is not possible. 

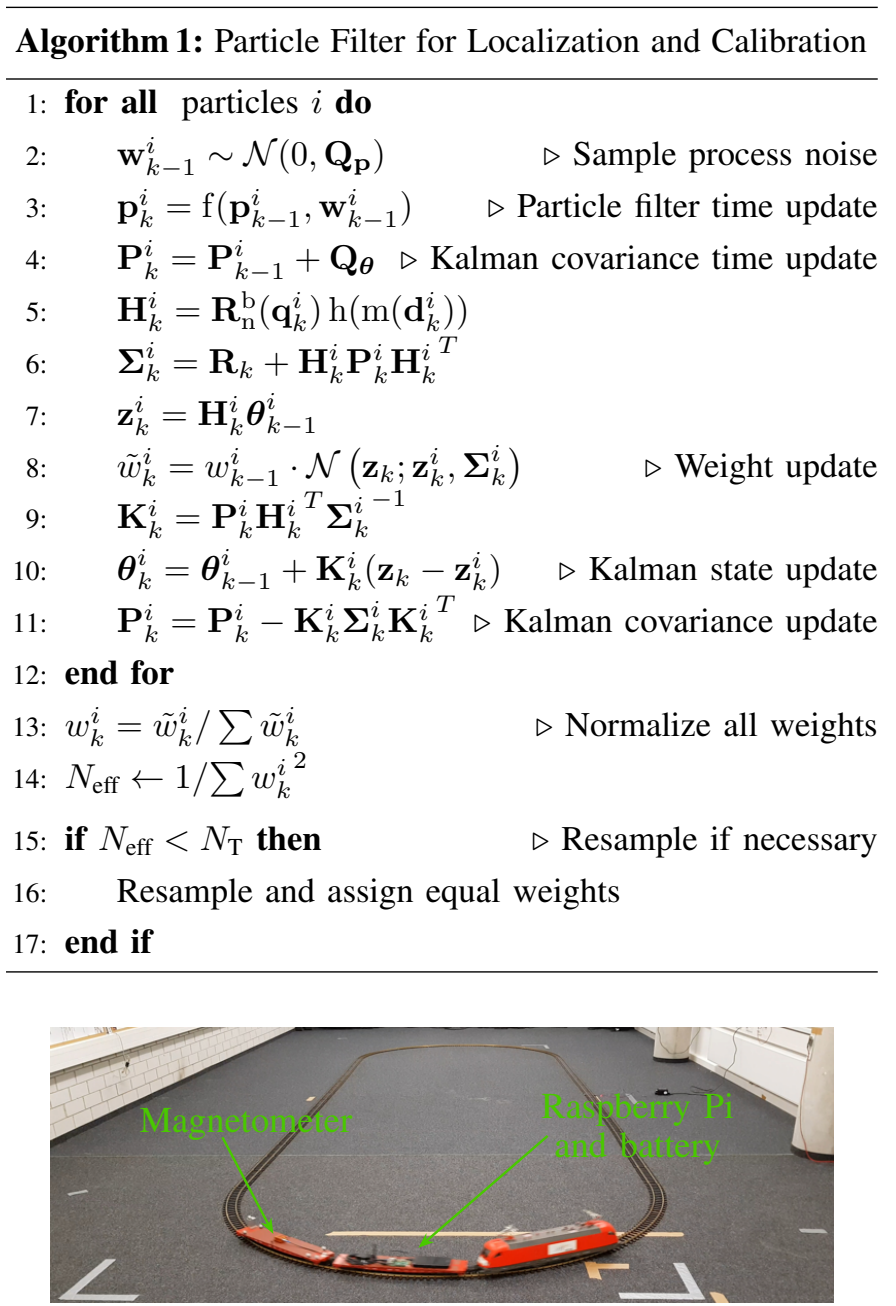

Fig. 1. Train track and the model train used to record the measurements. The track has an length of $\approx 17 \mathrm{~m}$. The first wagon of the model train carries a Raspberry Pi and a battery. On the second wagon the magnetometer triad is mounted.

\section{A. Measurement Setup}

For the evaluation, experiments with a model train in a laboratory were conducted. The model train was equipped with an Xsens MTi-G-700 IMU containing a triad of magnetometers. The magnetometers have a resolution and noise density of $19.53 \mathrm{nT}$ and $20 \mathrm{nT} / \sqrt{\mathrm{H} z}$ which is comparable to typical low-cost sensors. The measurements were recorded at a rate of $100 \mathrm{~Hz}$. The track of the model train is placed on the laboratory floor as can be seen in Fig. 1 and has a length of about $17 \mathrm{~m}$. The model train consists of a traction unit and two wagons. On the first wagon a Raspberry Pi logging the magnetometer data, a battery and a Wi-Fi module is installed. On the second wagon only the sensor is mounted. Additionally, a notebook computer logs the ground truth position of the sensor with a rate of $100 \mathrm{~Hz}$. The ground truth is obtained from an optical Vicon tracking system with sub-centimeter accuracy. The wagon itself is plastic and therefore has no influence on the calibration. To evaluate the calibration, ferromagnetic material was mounted on the wagon as shown in Fig. 2. First, a steal plate with nuts was placed below the sensor and then successively screws were added to all sides and finally a

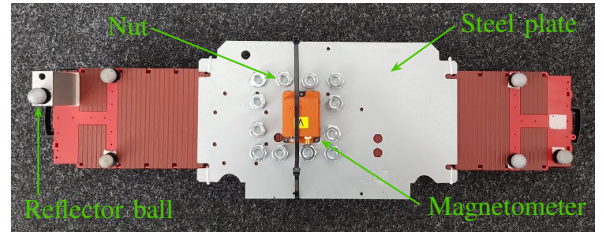

(a)

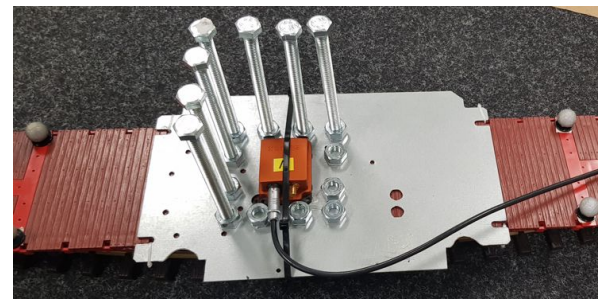

(b)

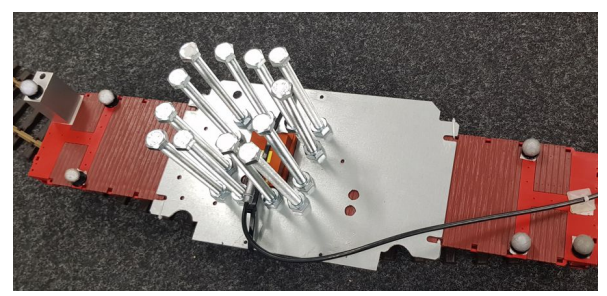

(c)

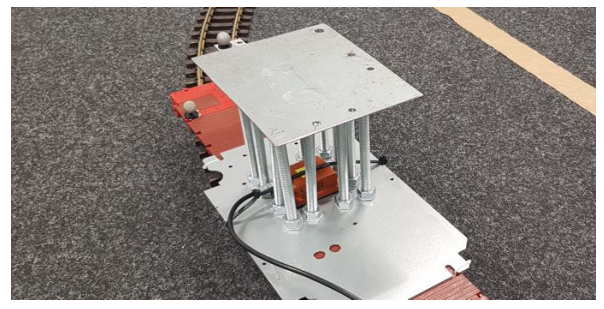

(d)

Fig. 2. Wagon that carries the sensor, a steel plate and an assembly of nuts. On the corner six infrared reflector balls are placed for position tracking with the Vicon system. (a) In the first setup the sensor is mounted on a steel plate with nuts on it. (b) In the second setup on two sides of the sensor steel screws are added. (c) In the third setup the sensor was completely surrounded by screws. (d) In the last setup in addition a steel plate was mounted on top of the screws.

second steel plate was placed on top of the screws. In total we measured with four different setups: sensor on steel plate, sensor on steel plate with screws on two, and four sides and the last setup contains screws on all sides and a second steel plate on top. During the measurements the train was driving with $\approx 1 \mathrm{~m} / \mathrm{s}$, which was the highest speed possible.

\section{B. Filter Implementation}

When the platform, here a train, moves on a known trajectory or track, it is useful to use this knowledge directly in the estimation to reduce the dimension of the state space and thus reducing the computational complexity. In the following the used coordinate system and state space model is introduced.

1) Track Coordinates: The train can only move along a specific track. The position of the train therefore can be described relative to the track. Formally the position is uniquely defined with the tuple $\left(s, n_{\text {track }}\right)$ containing the one dimensional along- 
track position $s \in \mathbb{R}_{+}$on track $n_{\text {track }} \in \mathbb{N}$. If there is only one track $s$ alone is already unique. Position $s$ is defined by the length of the track between the current train position and the starting point of the track. The track is assumed to be known, which is a reasonable assumption because trains and path following robots move on a predefined series of tracks.

2) State Space Model: The platform motion model is set to a piecewise constant Wiener acceleration model according to [31]. The state vector is

$$
\mathbf{p}_{k}=\left[\begin{array}{lll}
s_{k} & \dot{s}_{k} & \ddot{s}_{k}
\end{array}\right]^{T}
$$

and the system equations are

$$
\mathbf{p}_{k}=\left[\begin{array}{ccc}
1 & T & \frac{1}{2} T^{2} \\
0 & 1 & T \\
0 & 0 & 1
\end{array}\right] \mathbf{p}_{k-1}+\mathbf{w}_{k}^{p}
$$

with the time discrete covariance matrix of the process noise

$$
\mathbf{Q}_{k}=\left[\begin{array}{ccc}
\frac{1}{4} T^{4} & \frac{1}{2} T^{3} & \frac{1}{2} T^{2} \\
\frac{1}{2} T^{3} & T^{2} & T \\
\frac{1}{2} T^{2} & T & 1
\end{array}\right] \sigma_{w}^{2} .
$$

In (24) $\sigma_{w}$ has to be chosen depending on the maximum acceleration of the platform. A rule of thumb can be found in [31]. In principle it would be possible to also estimate the orientation of the magnetometer in relation to the track [19]. Here the orientation was assumed to be known to simplify the measurement model to

$$
\mathbf{z}_{k}=\mathrm{h}\left(\mathrm{m}\left(\mathbf{s}_{k}\right)\right) \boldsymbol{\theta}_{k}+\mathbf{n}_{k} .
$$

\section{Alternating Magnetic Field from Power Line}

The model train is powered with $230 \mathrm{~V} \mathrm{AC}$ at $50 \mathrm{~Hz}$ over a transformer that reduces the voltage to $16 \mathrm{~V}$. This voltage is applied to the track. Depending on the current that the engine requires, a magnetic field alternating with the $50 \mathrm{~Hz}$ frequency of the voltage is induced. Since the alternating field is not location dependent, this signal can be seen as a disturbance that negatively affects the estimation. To remove this noise a simple moving average low-pass filter was applied that averages over the last two samples obtained from the magnetometer. This was possible because the frequency of the observed static magnetic field is well below $50 \mathrm{~Hz}$ due to the low train speed. If this is not the case but the frequency of the disturbance is known a band-stop filter can be used instead or the amplitude and phase of the disturbance can be estimated in the particle filter.

\section{Start Detector}

When the calibration parameters are not already estimated the particle filter can diverge when the train is not running. This is due to the limited number of particles and the fact that in standstill no particle can estimate meaningful calibration parameters due to a lack of observability. To avoid this behavior a detector was implemented to detect when the train starts to drive based on the magnetometer measurements. The detector uses the well known cumulative sum algorithm [32] that detects abrupt changes in the mean of a sequence of measurements.

\section{E. Map Creation}

The evaluation of the map is performed for each particle and every measurement (Algorithm 1 Line 5) and hence should have a low complexity. In the evaluation therefore the map is reduced to an array containing only the magnetic field at discrete positions. The spacing of the along-track positions is $\Delta s=1 \mathrm{~cm}$. The access to the map is performed with an index calculation $i_{s}=\operatorname{round}\left(\frac{s}{\Delta s}\right)$ and a look-up in the array. For the evaluation the map was extended to contain the 3-D positions of the discrete along-track positions. This simplifies the calculation of the position error. The actual map creation uses multiple runs over the track and consists of two steps. First, for each measured magnetic field vector the position provided by the Vicon system is matched to the closest discrete along track position. Second, a kernel regression is performed to ensure a smooth map and to remove noise from the sensor and the $50 \mathrm{~Hz}$ power supply.

In this example the mapping process was straightforward due to the availability of the ground truth positions. To obtain such a map in practice one can use SLAM methods [11] or a reference platform with additional sensors like lidars, cameras, IMUs and odometers. The benefit of the proposed approach is that due to the simultaneous calibration the map has to be created only once and then can be used by multiple platforms equipped only with low-cost magnetometers. The map itself will be subject to changes over time due to a slowly changing earth magnetic field [33] and changes in the environment, e.g., due to construction work. While the changes in the earth magnetic field in one year are relatively small, typically below $1 \%$, compared to the static variations observed in our lab and railway measurements [17], the changes due to the environment can be severe and hence require an update of the map. Temporal changes or distortions in the magnetic field caused, e.g., by power lines have to be accounted for in the localization algorithm by filtering specific frequencies or by robust estimation filters [34].

\section{F. Results}

In the evaluation a total of 16 combinations of maps and datasets were processed. To get the 16 combinations, four maps were created with the measurements of the different setups in Fig. 2 and the corresponding ground truth positions. Each dataset was then used for estimating the position and calibration based on the four maps. The magnetic field in each map is different due to the different calibration parameters caused by the different setups. Therefore the estimated parameters of the 16 combinations are also different. Since the particle filter is a stochastic filter, 100 Monte-Carlo runs for each of the combinations were performed to verify that the results are stable. The number of particles was set to 5000, which allowed the filter to run in real time on a notebook processor. The positions of the initial particles are placed equidistant within a $3 \mathrm{~m}$ interval around the ground truth position. The initial speed and acceleration is sampled from 
TABLE I

ENERGY AND VARIANCE OF THE DATASETS

\begin{tabular}{|c|c|c|c|c|}
\hline & dataset & dataset & dataset 3 & dataset 4 \\
\hline Variance & 0.266 & 0.211 & 0.184 & 0.182 \\
\hline Energy & 5022 & 19487 & 12737 & 11966 \\
\hline
\end{tabular}

uniform distributions in the intervals $\pm 1 \mathrm{~m} / \mathrm{s}$ and $\pm 0.5 \mathrm{~m} / \mathrm{s}^{2}$. The Kalman filters are initialized like the sensor is already calibrated hence the bias vector is set to zero and the matrix $\mathbf{C}$ in (2) is set to an identity matrix. The initial covariance of the bias is $2^{2}$ and for the rest of the parameters $1^{2}$. The datasets were truncated to a common duration of $4.75 \mathrm{~min}$ after the detection of the start to ensure comparability of the results. During the measurements the model train was accelerating constantly from standstill to its maximum speed and then kept driving.

To show the accuracy of the estimated position the rootmean-square error (RMSE) was calculated for all runs and combinations. The RMSE is calculated from the 3-D position ground truth and the 3-D position estimated by the particle filter. The estimated 3-D position is obtained from a look-up in the map at the index $i_{s}=\operatorname{round}\left(\frac{\hat{s}}{\Delta s}\right)$ with the MMSEE $\hat{s}$ of the along-track position given by

$$
\hat{s}_{k}=\sum_{i=1}^{N_{p}} w_{k}^{i} s_{k}^{i} .
$$

The 3-D position error $\epsilon_{\mathrm{pos}}$ is

$$
\boldsymbol{\epsilon}_{\mathrm{pos}}=\left[\begin{array}{lll}
\delta x & \delta y & \delta z
\end{array}\right]^{T}=\left[\begin{array}{lll}
x-\hat{x} & y-\hat{y} & z-\hat{z}
\end{array}\right]^{T}
$$

with the ground truth position vector $\left[\begin{array}{lll}x & y & z\end{array}\right]^{T}$ and the estimated position vector $\left[\begin{array}{lll}\hat{x} & \hat{y} & \hat{z}\end{array}\right]^{T}$ found from $\hat{s}$. Therefore the RMSE is

$$
\bar{\epsilon}_{\mathrm{pos}}=\sqrt{\frac{1}{K} \sum_{k=0}^{K-1}\left(\delta x_{k}^{2}+\delta y_{k}^{2}+\delta z_{k}^{2}\right)}
$$

with the number of time steps $K$. The box plot in Fig. 3 shows the statistics of the RMSE over all Monte-Carlo runs and the 16 combinations. The lower and upper whiskers are the minimal and the maximal RMSE, the horizontal line in the box is the median, and the borders of the boxes are the $25 \%$ and $75 \%$ quantiles. The numbering of the datasets reflects the order of the measurement setups in Fig. 2. For each data set the different colors are the different maps where blue is the map created with the setup in Fig. 2a, red with Fig. 2b and so on. The results show a high accuracy of a few centimeters. Overall the RMSE was always below $6 \mathrm{~cm}$. The RMSE values of the different maps for the same dataset are relatively close to each other, this is a first indicator that the calibration is working correctly. In contrast to this, a bigger difference between different datasets can be observed. One property of the dataset that seems to be connected to the achievable RMSE is the variance shown in Table I. The table shows the trace of the sample covariance matrix and the signal energy of the datasets.

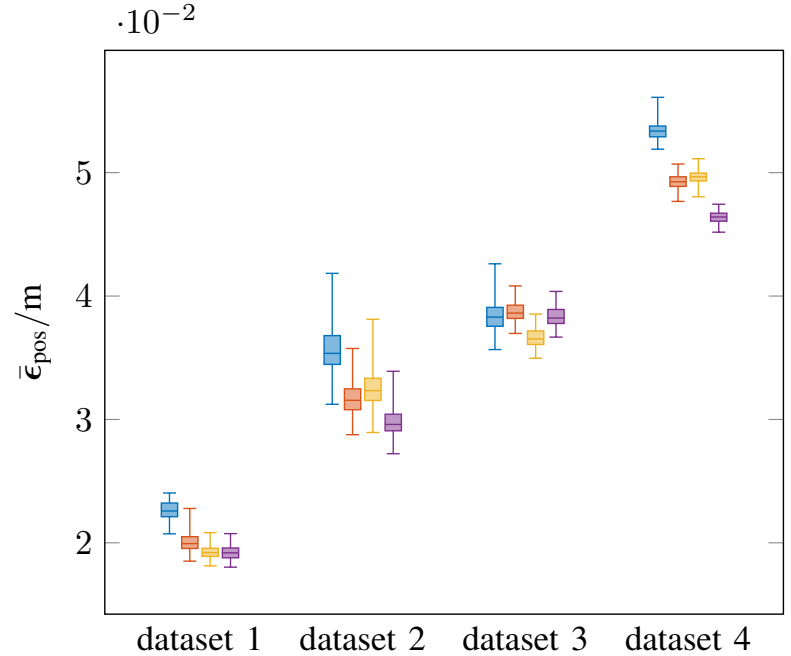

Fig. 3. Statistics of the particle filter position RMSE in meters for the 100 Monte-Carlo runs and the 16 different combinations.
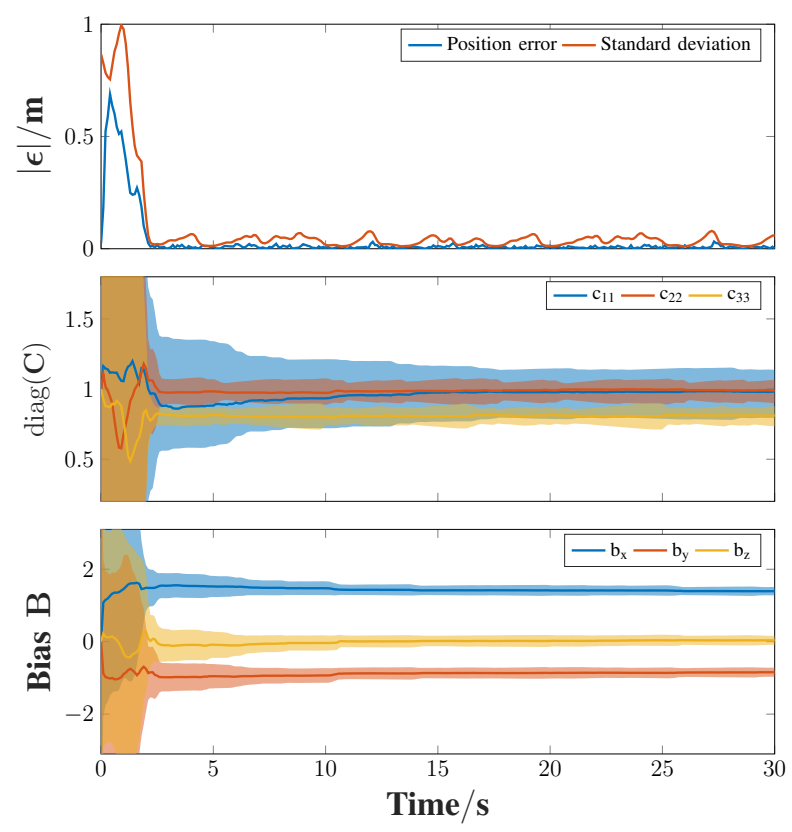

Fig. 4. (top) Absolute 3-D position error of the particle filter estimate and standard deviation of the point cloud in meters. The example is obtained with dataset 2 recorded with setup Fig. $2 \mathrm{~b}$ and a map recorded with Fig. 2a. (middle) Kalman filter estimate (29) of the diagonal elements of matrix $\mathbf{C}$ from (2). The colored area is three times the estimated standard deviation (30). (bottom) Kalman filter estimate (29) of the bias vector $\mathbf{b}$ from (2). The colored area is three times the estimated standard deviation (30).

While a higher variance of the measured magnetic field leads to a smaller RMSE in Fig. 3, the signal energy does not show a clear influence on the RMSE. Intuitively this is reasonably, the signal energy accounts also for the mean and therefore the bias. The bias increases the energy but does not contribute any position information because it is constant for all positions. The temporal behavior of the position error and the parameter estimation is shown in Fig. 4 for one example. The middle and bottom part show the estimated diagonal elements of matrix $\mathbf{C}$ and the bias $\mathbf{b}$ of (2). The diagonal elements of $\mathbf{C}$ can be interpreted as scaling factors between the map and the dataset 
when the off-diagonal elements are close to zero, which was the case in this example. The MMSEE of the parameters $\hat{\boldsymbol{\theta}}$ in Fig. 4 and the corresponding covariance matrix $\hat{\mathbf{P}}_{k}$ is given by a mixture of all Kalman filter estimates and the particle weights after the update step [35, p. 48]

$$
\begin{aligned}
\hat{\boldsymbol{\theta}}_{k} & =\sum_{i=1}^{N_{p}} w^{i} \boldsymbol{\theta}_{k}^{i} \\
\hat{\mathbf{P}}_{k} & =\sum_{i=1}^{N_{p}} w_{k}^{i}\left[\mathbf{P}_{k}^{i}+\left(\boldsymbol{\theta}_{k}^{i}-\hat{\boldsymbol{\theta}}_{k}\right)\left(\boldsymbol{\theta}_{k}^{i}-\hat{\boldsymbol{\theta}}_{k}\right)^{T}\right] .
\end{aligned}
$$

Note that the initial position error in the top part of Fig. 4 is close to zero because the particles are initially placed equally spaced in a $3 \mathrm{~m}$ interval around the true position with equal weights. This does not mean the filter knows the exact position as can be seen from the standard deviation of the position that is calculated over all particles in the point cloud. In the beginning the error increases quickly due to a high uncertainty in the calibration parameters, indicated by the colored areas in the middle and bottom part of Fig. 4. When more measurements become available and the train has moved further the calibration parameters become observable. This reduces the estimated uncertainty in the Kalman filters and particles on a wrong trajectory with wrongly estimated calibration parameters are assigned low weights in the particle filter update step and hence are not resampled. This can be seen from the position standard deviation. The standard deviation decreases and therefore the particle cloud shrinks until it only spans a few centimeters around the true position. Evaluating the quality of the magnetometer calibration is not straightforward because the true calibration parameters are unknown. The quality is therefore judged by how close the calibrated map data obtained from the measurement model (25) is to the sensor readings. In Fig. 5 the magnetic field vector from the map before and after calibration, and the measurements are shown. The measured magnetic field is normalized internally by the sensor with a constant value. According to the data sheet of the sensor manufacturer the normalization constant is $\approx 40 \mu \mathrm{T}$. The magnetic field of the map is obtained from the map and the ground truth positions. In the beginning the calibration is not estimated properly but after a view seconds the estimation converges towards the correct value and the calibrated map data nicely fits to the sensor readings. This observation is in correspondence with Fig. 4 where we see that the calibration converged after roughly the same time. Furthermore, a comparison of the bias and scale factor estimates in Fig. 4 with the value in Fig. 5 shows the estimates converge to the correct values. From Fig. 4 and Fig. 5 it becomes clear that the most challenging part is the initialization. In the initialization phase it is important that the train or in general the platform starts and keeps driving with a trajectory that fits the movement model with a smoothly changing acceleration and platform attitude. This ensures the observability and quick convergence of the calibration parameters and that the particle cloud can follow the correct trajectory. If the calibration parameters are not converging after a few seconds or the train is performing some unexpected
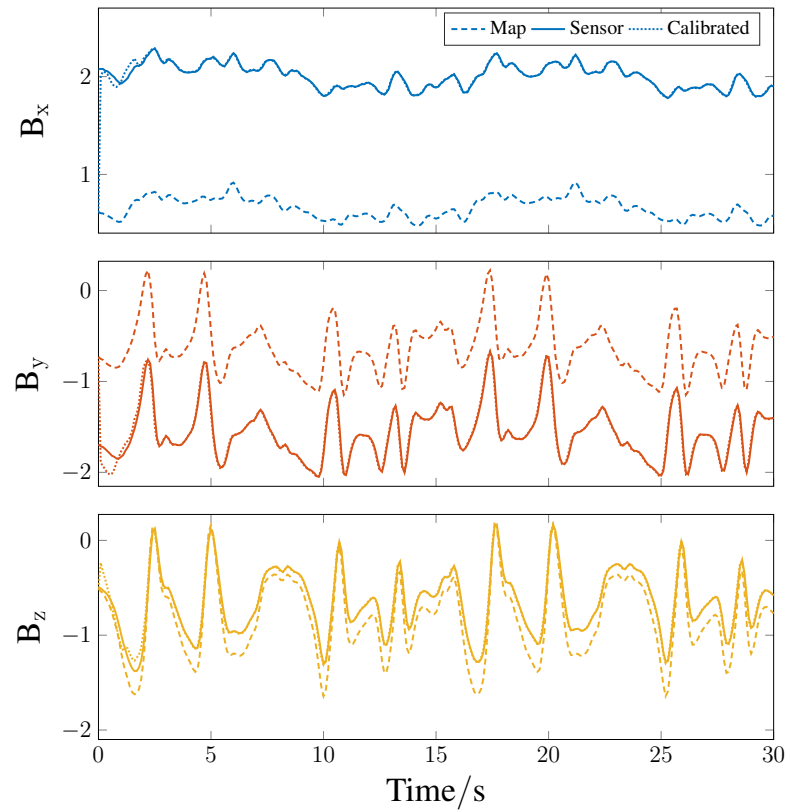

Fig. 5. Calibration result of the particle filter for the dataset 2 recorded with the setup in Fig. $2 b$ and a map recorded with Fig. 2a. The colors fit the colors of the parameter estimation in Fig. 4. The figure shows the raw measurements (solid line) of the $\mathrm{x}^{-}, \mathrm{y}$ - and $\mathrm{z}$-axis of the magnetometer and the corresponding magnetic map values (dashed line). The dotted line is the expected measurement from (25) calculated with the magnetic vector from the map at the ground truth position and the estimated calibration parameters (29). The magnetic field is unitless due to the internal normalization of the magnetometer. According to the sensor manufacture a sensor output of 1 corresponds approximately to $40 \mu \mathrm{T}$.

movement, the point cloud keeps spreading out and it is becoming unlikely that a particle follows the right trajectory. In combination with the resampling step then the particle set most likely degenerates. This problem is caused by the limited number of particles that introduces the need for resampling and by considering only the measurements of the uncalibrated magnetometer. If additional sensors like an odometer or IMU are incorporated into the prediction step of the filter, the particles can follow also more dynamic trajectories and the overall initialization phase should become more robust.

In Fig. 6 the energy of the calibration error is given in relation to the signal energy of the measured magnetic field $\mathbf{z}_{k}$ from which its mean $\overline{\mathbf{z}}$ was removed

$$
\overline{\boldsymbol{\epsilon}}_{\mathrm{cal}}=\sum_{k=0}^{K-1}\left\|\hat{\mathbf{z}}_{k}-\mathbf{z}_{k}\right\|_{2}^{2} / \sum_{k=0}^{K-1}\left\|\mathbf{z}_{k}-\overline{\mathbf{z}}\right\|_{2}^{2},
$$

where $\|\cdot\|_{2}$ is the Euclidean norm, $K$ is the number of time steps and $\hat{\mathbf{z}}_{k}$ is the calibrated magnetic field vector. The calibrated magnetic field vector is obtained from (25), the estimated calibration parameters $\hat{\boldsymbol{\theta}}_{k}$ from (29) and the magnetic field in the map at the ground truth position. The mean was removed for better comparison of the results. If simply the signal energy would be used, datasets with a large bias would achieve smaller values compared to datasets with a smaller bias even though the error energy is the same. The box plot in Fig. 6 is calculated over the different Monte-Carlo runs and has the same color coding and shows the same statistical quantities for $\overline{\boldsymbol{\epsilon}}_{\mathrm{cal}}$ as Fig. 3 does for the position RMSE. The 


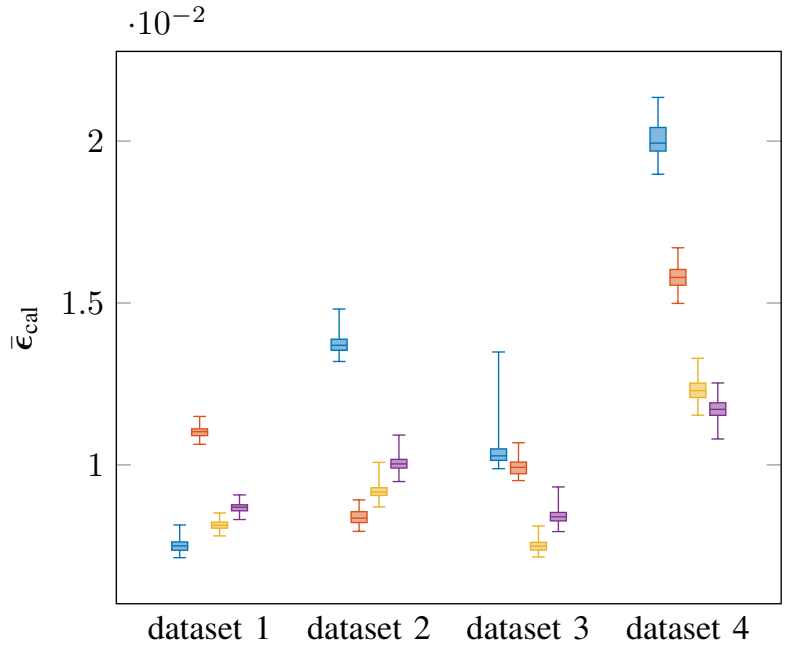

Fig. 6. Statistics of the relative error $\overline{\boldsymbol{\epsilon}}_{\mathrm{cal}}$ of the calibrated map data for the 100 Monte-Carlo runs and the 16 different datasets. The error is unitless due to the internal normalization of the magnetometer.

statistics in Fig. 6 show that the signal energy of the error is two orders of magnitude smaller than the energy of the measured magnetic field. In analogy to the signal to noise ratio used in communications, this means that the signal to error ratio is always higher than $16 \mathrm{~dB}$. To get a better impression of how the calibration reduces the error between the map and the measured magnetic field, Table II shows the calibration gain. The gain is defined by the ratio of the error energy between the measurements and the map before and after calibration

$$
g_{\text {cal }}=\sum_{k=1}^{K-1}\left\|\mathbf{z}_{k}^{n}-\mathbf{z}_{k}\right\|_{2}^{2} / \sum_{k=1}^{K-1}\left\|\hat{\mathbf{z}}_{k}-\mathbf{z}_{k}\right\|_{2}^{2}
$$

where $\mathbf{z}_{k}^{n}$ is the magnetic field directly obtained from the magnetic map without calibration. The gain in Table II is the gain achieved with the worst calibration result from the different Monte-Carlo runs. Therefore the gain is always higher than 84.27 and up to 843.24 when the map and the dataset was not recorded with the same setup. The table also shows that the calibration is close to the uncalibrated dataset when the same setup was used for the map creation than for localization. In one case it is even better which can be explained by the fact that the map can contain small systematic errors due to sensor and remaining $50 \mathrm{~Hz}$ noise. The Kalman filter can adapt the calibration to these small errors and therefore the result can be slightly better. Combined with the low RMSE for the position in Fig. 3, the results for the calibration are a strong indicator that the proposed method works and high position and calibration accuracy is attainable considering only the magnetometer readings.

\section{CONCLUSION}

In this paper, a method for simultaneous localization and magnetometer calibration with local magnetic field distortions was introduced. This method enables magnetometer calibration also for heavy platforms, where common calibration methods are not applicable. The proposed method is based on a Rao-Blackwellized particle filter that estimates the pose of the
TABLE II

WORST CASE CALIBRATION GAIN

\begin{tabular}{|c|c|c|c|c|}
\hline nap 1 & 0.91 & 828.56 & 361.73 & 296.79 \\
\hline nap 2 & 843.24 & 1.07 & 370.30 & 273.90 \\
\hline nap 3 & 397.62 & 345.36 & 0.83 & 84.27 \\
\hline nap 4 & 481.97 & 361.67 & 120.49 & 0.65 \\
\hline
\end{tabular}

platform with a particle filter and the calibration parameters with a Kalman filter. The Rao-Blackwellization reduces the computational complexity and allows the algorithm to run in real time. The algorithm was formulated for a generic, nonlinear state space model to allow an easy adaptation to different applications. In an evaluation the feasibility of the proposed method was shown based on measurements recorded with a model train and four different measurement setups. In the evaluation the filter was capable to accurately estimate the position and calibration parameters. The position error was within a few centimeters and the calibration error had an energy two orders of magnitude smaller than the measured magnetic field. The evaluated filter used only magnetometer measurements but the filter is easily extendable with an odometer or an IMU to improve the robustness in the initialization phase when the calibrations parameters are still unknown. For applications with more degrees of freedom and a fast changing platform attitude an IMU should be used in the estimation. This will improve the performance and lower the number of required particles.

\section{REFERENCES}

[1] I. Skog, P. Handel, J.-O. Nilsson, and J. Rantakokko, "Zero-Velocity Detection-An Algorithm Evaluation," IEEE Transactions on Biomedical Engineering, vol. 57, no. 11, pp. 2657-2666, Nov. 2010.

[2] F. Gustafsson and F. Gunnarsson, "Mobile positioning using wireless networks: possibilities and fundamental limitations based on available wireless network measurements," IEEE Signal Processing Magazine, vol. 22, no. 4, pp. 41-53, Jul. 2005.

[3] C. Gentner, T. Jost, W. Wang, S. Zhang, A. Dammann, and U.-C. Fiebig, "Multipath Assisted Positioning with Simultaneous Localization and Mapping," IEEE Transactions on Wireless Communications, vol. 15, no. 9, pp. 6104-6117, Sep. 2016.

[4] B. Gozick, K. P. Subbu, R. Dantu, and T. Maeshiro, "Magnetic Maps for Indoor Navigation," IEEE Transactions on Instrumentation and Measurement, vol. 60, no. 12, pp. 3883-3891, Dec. 2011.

[5] B. Li, T. Gallagher, A. G. Dempster, and C. Rizos, "How feasible is the use of magnetic field alone for indoor positioning?" in 2012 International Conference on Indoor Positioning and Indoor Navigation (IPIN), Nov. 2012, pp. 1-9.

[6] M. Frassl, M. Angermann, M. Lichtenstern, P. Robertson, B. J. Julian, and M. Doniec, "Magnetic maps of indoor environments for precise localization of legged and non-legged locomotion," in 2013 IEEE/RSJ International Conference on Intelligent Robots and Systems, Nov. 2013, pp. 913-920.

[7] W. Shao, F. Zhao, C. Wang, H. Luo, T. Muhammad Zahid, Q. Wang, and D. Li, "Location Fingerprint Extraction for Magnetic Field Magnitude Based Indoor Positioning," Journal of Sensors, 2016.

[8] S. Lee, Y. Chon, and H. Cha, "Smartphone-Based Indoor Pedestrian Tracking Using Geo-Magnetic Observations," Mobile Information Systems, vol. 9, no. 2, pp. 123-137, 2013.

[9] J. Haverinen and A. Kemppainen, "Global indoor self-localization based on the ambient magnetic field," Robotics and Autonomous Systems, vol. 57, no. 10, pp. 1028-1035, Oct. 2009. 
[10] J. Jung, S. M. Lee, and H. Myung, "Indoor Mobile Robot Localization and Mapping Based on Ambient Magnetic Fields and Aiding Radio Sources," IEEE Transactions on Instrumentation and Measurement, vol. 64, no. 7, pp. 1922-1934, Jul. 2015.

[11] M. Kok and A. Solin, "Scalable Magnetic Field SLAM in 3D Using Gaussian Process Maps," in 2018 21st International Conference on Information Fusion (FUSION), Jul. 2018, pp. 1353-1360.

[12] J. A. Shockley and J. F. Raquet, "Navigation of Ground Vehicles Using Magnetic Field Variations," Navigation, vol. 61, no. 4, pp. 237-252, 2014. [Online]. Available: https://onlinelibrary.wiley.com/doi/abs/10.1002/navi.70

[13] J. A. Shockley, "Ground Vehicle Navigation Using Magnetic Field Variation," Ph.D. dissertation, Sep. 2012. [Online]. Available: https://apps.dtic.mil/docs/citations/ADA564651

[14] A. Canciani and J. Raquet, "Airborne Magnetic Anomaly Navigation," IEEE Transactions on Aerospace and Electronic Systems, vol. 53, no. 1, pp. 67-80, Feb. 2017.

[15] H. Zhang and F. Martin, "Robotic mapping assisted by local magnetic field anomalies," in 2011 IEEE Conference on Technologies for Practical Robot Applications, Apr. 2011, pp. 25-30.

[16] E. Le Grand and S. Thrun, "3-Axis magnetic field mapping and fusion for indoor localization," in 2012 IEEE International Conference on Multisensor Fusion and Integration for Intelligent Systems (MFI), Sep. 2012, pp. 358-364.

[17] O. Heirich, B. Siebler, and E. Hedberg, "Study of Train-Side Passive Magnetic Measurements with Applications to Train Localization," Journal of Sensors, 2017.

[18] B. Siebler, O. Heirich, and S. Sand, "Bounding INS Positioning Errors with Magnetic-Field-Signatures in Railway Environments," in Proceedings of the 30th International Technical Meeting of The Satellite Division of the Institute of Navigation (ION GNSS+ 2017), 2017, pp. 3224-3230.

[19] — , "Train Localization with Particle Filter and Magnetic Field Measurements," in 2018 21st International Conference on Information Fusion (FUSION), Jul. 2018, pp. 1-5.

[20] J. F. Vasconcelos, G. Elkaim, C. Silvestre, P. Oliveira, and B. Cardeira, "Geometric Approach to Strapdown Magnetometer Calibration in Sensor Frame," IEEE Transactions on Aerospace and Electronic Systems, vol. 47, no. 2, pp. 1293-1306, Apr. 2011.

[21] V. Renaudin, M. H. Afzal, and G. Lachapelle, "Complete Triaxis Magnetometer Calibration in the Magnetic Domain," Journal of Sensors, 2010.

[22] R. Alonso and M. D. Shuster, "Complete Linear Attitude-Independent Magnetometer Calibration," Journal of the Astronautical Sciences, vol. 50, no. 4, pp. 477-490, 2002.

[23] M. Kok and T. B. Schön, "Magnetometer Calibration Using Inertial Sensors," IEEE Sensors Journal, vol. 16, no. 14, pp. 5679-5689, Jul. 2016.

[24] A. Wahdan, J. Georgy, and A. Noureldin, "Three-Dimensional Magnetometer Calibration With Small Space Coverage for Pedestrians," IEEE Sensors Journal, vol. 15, no. 1, pp. 598-609, Jan. 2015.

[25] H. L. Van Trees and K. L. Bell, Bayesian Bounds for Parameter Estimation and Nonlinear Filtering/Tracking. Wiley-IEEE Press, 2007. [Online]. Available: https://ieeexplore.ieee.org/book/5263120

[26] T. Schon, F. Gustafsson, and P. Nordlund, "Marginalized particle filters for mixed linear/nonlinear state-space models," IEEE Transactions on Signal Processing, vol. 53, no. 7, pp. 2279-2289, Jul. 2005.

[27] K. Murphy and S. Russell, "Rao-Blackwellised Particle Filtering for Dynamic Bayesian Networks," in Sequential Monte Carlo Methods in Practice, A. Doucet, N. Freitas, and N. Gordon, Eds. New York, NY: Springer New York, 2001, pp. 499-515.

[28] M. Montemerlo, S. Thrun, D. Koller, and B. Wegbreit, "FastSLAM: A Factored Solution to the Simultaneous Localization and Mapping Problem," in National Conference on Artificial Intelligence (AAAI-02), 2002.

[29] M. S. Arulampalam, S. Maskell, N. Gordon, and T. Clapp, "A tutorial on particle filters for online nonlinear/non-Gaussian Bayesian tracking," IEEE Transactions on Signal Processing, vol. 50, no. 2, pp. 174-188, Feb. 2002.

[30] S. Thrun, W. Burgard, and D. Fox, Probabilistic robotics. MIT press, 2005.

[31] Y. Bar-Shalom and X.-R. Li, Estimation and Tracking: Principles, Techniques, and Software. Artech House, 1993.

[32] M. Basseville and I. V. Nikiforov, Detection of abrupt changes: theory and application. Prentice-Hall, Inc., 1993.

[33] I. Wardinski and R. Holme, "A time-dependent model of the Earth's magnetic field and its secular variation for the period 1980-2000,"
Journal of Geophysical Research: Solid Earth, vol. 111, no. B12, Dec. 2006.

[34] B. Siebler, O. Heirich, S. Sand, and U. D. Hanebeck, "Joint Train Localization and Track Identification based on Earth Magnetic Field Distortions," in 2020 IEEE/ION Position, Location and Navigation Symposium (PLANS), Apr. 2020, pp. 941-948.

[35] P.-J. Nordlund, "Sequential Monte Carlo Filters and Integrated Navigation," Licentiate thesis, Linköping University, 2002. [Online]. Available: http://urn.kb.se/resolve?urn=urn:nbn:se:liu:diva-98163

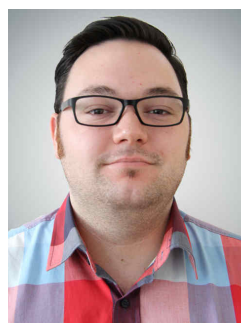

Benjamin Siebler (M'18) received his M.Sc. in electrical engineering and information technology from the Karlsruhe Institute of Technology (KIT), Germany, in 2014. He is currently pursuing his $\mathrm{PhD}$ at the Intelligent Sensor-Actuator-Systems Laboratory of the KIT. Since 2014 he is a researcher at the Institute of Communications and Navigation of the German Aerospace Center in Oberpfaffenhofen. His research focus is on multisensor fusion for localization applications with inertial sensors and magnetic field distortions.

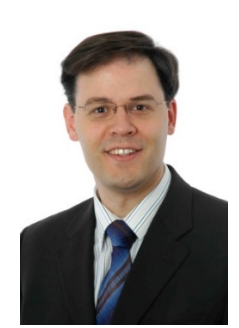

Stephan Sand (SM'10) received his $\mathrm{PhD}$ from ETH Zurich, Switzerland in 2010. Since 2002, Stephan has been researching on wireless communications and multi-sensor navigation at the Institute of Communications and Navigation of the German Aerospace Center in Oberpfaffenhofen, Germany Since 2014 he is leading the Vehicular Applications Group researching novel systems that combine robust navigation and wireless communications technologies for road users and railways. Stephan has authored and co-authored more than 100 publications in conferences and journal. He has obtained several patents and actively contributes to vehicular and railway communication standardization, e.g. in ETSI JTFIR and IEEE $802.11 \mathrm{bd}$

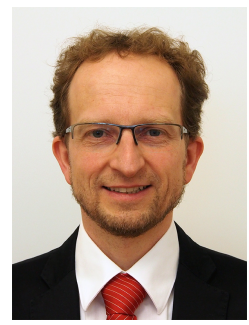

Uwe D. Hanebeck (F'17) is a chaired professor of Computer Science at the Karlsruhe Institute of Technology (KIT) in Germany and director of the Intelligent Sensor-Actuator-Systems Laboratory (ISAS). He obtained his Ph.D. degree in 1997 and his habilitation degree in 2003, both in Electrical Engineering from the Technical University in $\mathrm{Mu}$ nich, Germany. His research interests are in the areas of information fusion, nonlinear state estimation, stochastic modeling, system identification, and control with a strong emphasis on theory-driven approaches based on stochastic system theory and uncertainty models. He is author and coauthor of more than 500 publications in various high-ranking journals and conferences and an IEEE Fellow. 